\title{
Seizures in cancer patients: a vast spectrum of etiologies
}

\author{
Jad Costa*,1, Fady Gh Haddad² (D), Georges Costa1, Ahmad Harb¹, Roland Eid² (D), Hampig \\ Raphael Kourie ${ }^{2}$ \& Jeanine El Helou ${ }^{3}$ \\ ${ }^{1}$ Faculty of Medicine, Saint Joseph University, Beirut, Lebanon \\ ${ }^{2}$ Hematology \& Oncology Department, Faculty of Medicine, Saint Joseph University, Beirut, Lebanon \\ ${ }^{3}$ Neurology Department, Faculty of Medicine, Saint Joseph University, Beirut, Lebanon \\ *Author for correspondence: Tel.: +96 1793 17753; costajad@hotmail.com
}

\begin{abstract}
Aim: This study aims to recognize the distribution of different seizures etiologies in cancer patients, the most common primary tumors responsible for brain metastases, the most epileptogenic primary tumors and the therapeutic modalities. Methods: A retrospective study was conducted at Hotel-Dieu de France Hospital targeting patients admitted to hematology-oncology department between 2005 and 2016 who presented a seizure. Results: Of the 153 included patients, mean age was 57 years (standard deviation $=16$ years) and a male predominance $(66 \%)$. The majority of seizures were due to a primary tumor $(49 \%)$ or brain metastases (32\%). Other reversible (metabolic and drug) or nonreversible causes (carcinomatous meningitis, radiation and cardiovascular complications) formed the remaining causes. Regarding antiepileptic treatments, sodium valproate was mostly used, followed by levetiracetam and phenytoin. Conclusion: Numerous reversible causes are involved in the onset of epileptic seizures, including metabolic disorders, antibiotics and chemotherapies.
\end{abstract}

First draft submitted: 13 July 2019; Accepted for publication: 28 August 2019; Published online: 9 October 2019

Keywords: antiepileptic drugs $\bullet$ brain metastases $\bullet$ CNS neoplasms $\bullet$ epilepsy $\bullet$ gliomas $\bullet$ neuro-oncology $\bullet$ seizures

Seizures are a common manifestation of brain tumors, whether primary or secondary. Primary brain tumors can cause seizures early in the course of the disease [1] and are usually pharmacoresistant [2], whereas metastatic masses can also induce seizures, although less likely than primary brain tumors [3]. Brain metastases are the most common intracranial tumors in adults [4], originating from the lung, breast, skin (i.e., melanoma), kidney or colon with 70\% of these metastases annually originating from the lungs or breasts [5]. Small-cell lung cancer is the leading cause of brain metastases, followed by breast cancer and then melanoma [6-8]. The rate of seizures is not constant across all etiologies of brain neoplasms. For instance, the occurrence of epilepsy is highest in low-grade gliomas (60-85\%), followed by glioblastoma in 30-50\% of cases, and lowest in cerebral metastases, occurring in around a third of patients (20-35\%) [9].

In addition, cancer patients are at risk of seizures for a multitude of reasons unrelated to the tumor itself, such as cytotoxic chemotherapy, toxic metabolic encephalopathy, infectious processes and other rare causes such as neurological paraneoplastic syndromes, cranial irradiation and cerebrovascular complications [3]. Many cancer drugs at toxic doses can also cause seizures (cisplatin, busulphan, 5-fluorouracil, methotrexate and cyclosporine) [3]. Metabolic causes such as hyponatremia, hypocalcemia and hypomagnesaemia can also cause seizures. The treatment of the underlying disease or the discontinuation of the drug in question is usually enough to stop the seizures [3]. Cancer patients receiving treatment are more prone to CNS infections that can manifest as seizures [3]. Cranial irradiation can cause seizures either by acute radiation encephalopathy or by delayed radiation necrosis [10].

In addition to treating the underlying etiology, antiepileptic drugs (AEDs) are used to control seizures. Several factors affect the choice of AEDs in cancer patients; mainly drug interactions with cancer drugs via enzymatic induction (carbamazepine and phenytoin) [3,11], inhibition of enzymes in shared metabolic pathways (increased toxicity of phenytoin when combined with 5 -fluorouracil and decreased seizure control when combined with cisplatin) [12]. Other limiting factors are the route of administration and adverse effects (namely, hematologic) [3].

Future Medicine 


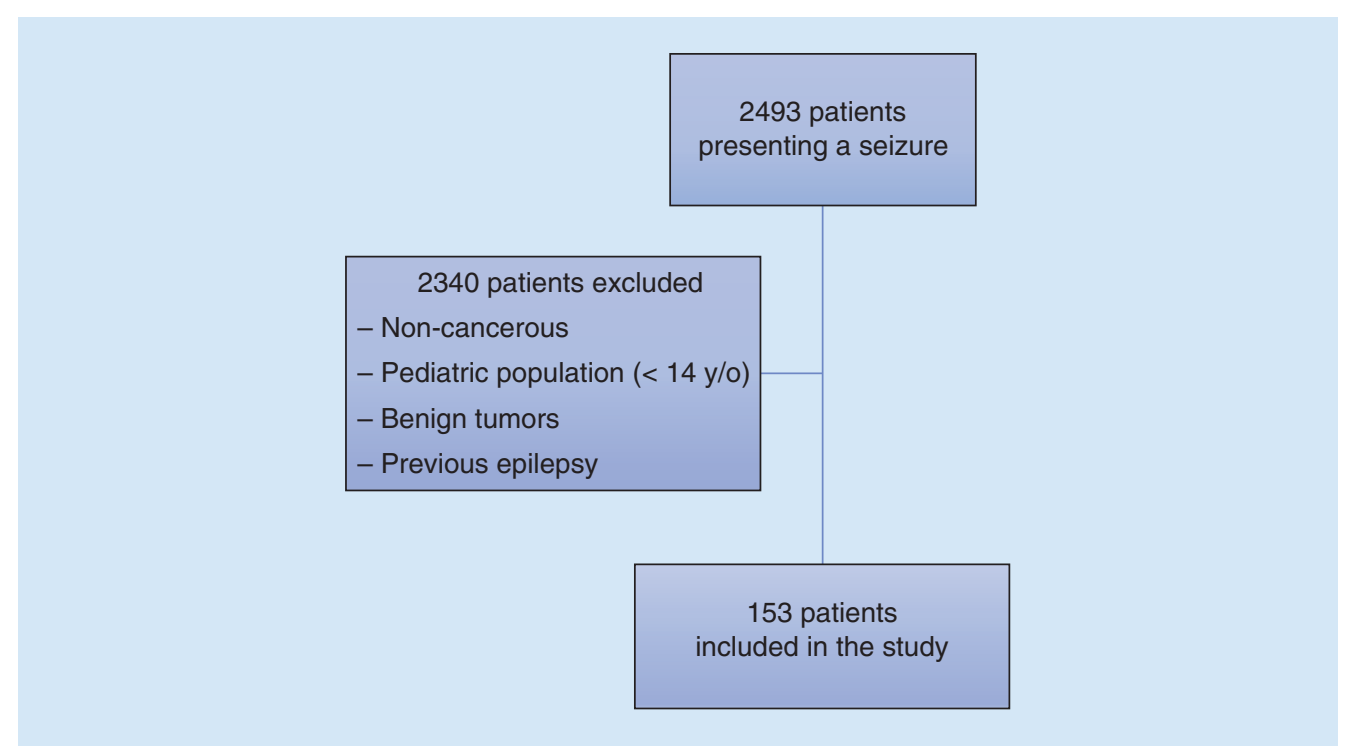

Figure 1. Selection of patients for analysis.

In this study we aimed to evaluate the various etiologies of seizures among cancer patients, differentiate between reversible and irreversible causes, and determine the AEDs used to treat seizures in our population in order to confirm what has been previously published and reproduce the data at our institution which allows us to better understand epilepsy among cancer patients for better management.

\section{Methods}

This is a retrospective study done on a population of hospital-admitted cancer patients who presented seizures during their disease course. This study was approved by the ethical committee at the Faculty of Medicine of Saint Joseph University. No informed consent was needed for this study.

We reviewed all patients who were hospitalized at Hotel-Dieu de France University Hospital, Beirut, Lebanon, between 2005 and 2016 and who had presented a seizure during their stay. Of those, we selected only patients that were known to have cancer. Those who had previous epilepsy and patients younger than 14 years were excluded (Figure 1).

We analyzed this group for the etiology of seizures which was determined by a neurologist (after going through the patient's case and ordering additional tests when necessary) in order to confirm the occurrence of the seizure and to determine the exact cause behind it (related to the tumor itself or due to other factors). The cases were divided into primary or secondary brain tumors, toxic or metabolic causes, iatrogenic causes - such as chemotherapy, radiation therapy or other medication - and cardiovascular causes. Patients were also evaluated for the type of cancer they had, and the type of AED they received. Variables were recorded as categorical or ordinal, and descriptive statistics were then performed using SPSS statistics version 25.0.

\section{Results}

Out of 2493 patients who were hospitalized and presented a seizure, 153 patients had cancer and were included in our study (Figure 1). The mean age of our population was 57 years (standard deviation $=16$ years) and a male predominance $(66 \%)$.

Regarding the etiologies of the seizures (Figure 2), primary tumors were incriminated in around half of cases (49\%), whereas brain metastases were the cause of seizures in around the third of cases $(32 \%)$. Other causes were less frequently implicated and were divided into reversible (drugs $2.6 \%$ and metabolic $2 \%$ ) and nonreversible (carcinomatous meningitis 3.3\%, cardiovascular complications $2.6 \%$ [cerebral bleeding or hematoma] and irradiation $1.3 \%)$. Other rare etiologies to mention are stereotactic biopsy complications $(1.3 \%)$, chemotherapy, locoregional invasion and multiorgan failure ( $0.7 \%$ each). Seizures remained of unknown origin in $3.1 \%$ of cases.

The most common types of cancer occurring in our studied population (Figure 3) were mainly primary brain tumors (50.3\%), lung cancer (19\%), breast cancer (9.8\%) and hematological malignancies (6.5\%). Less frequent 


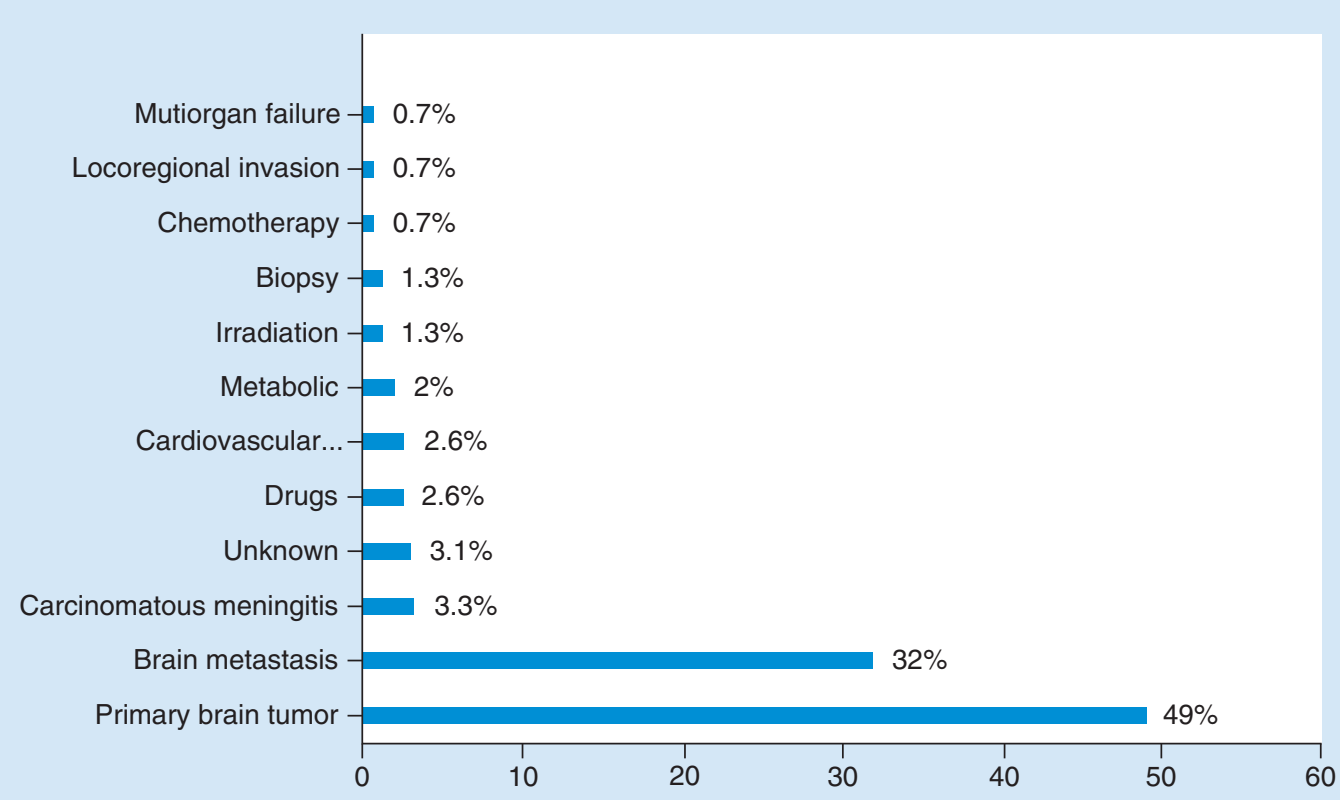

Figure 2. Etiologies of seizures.

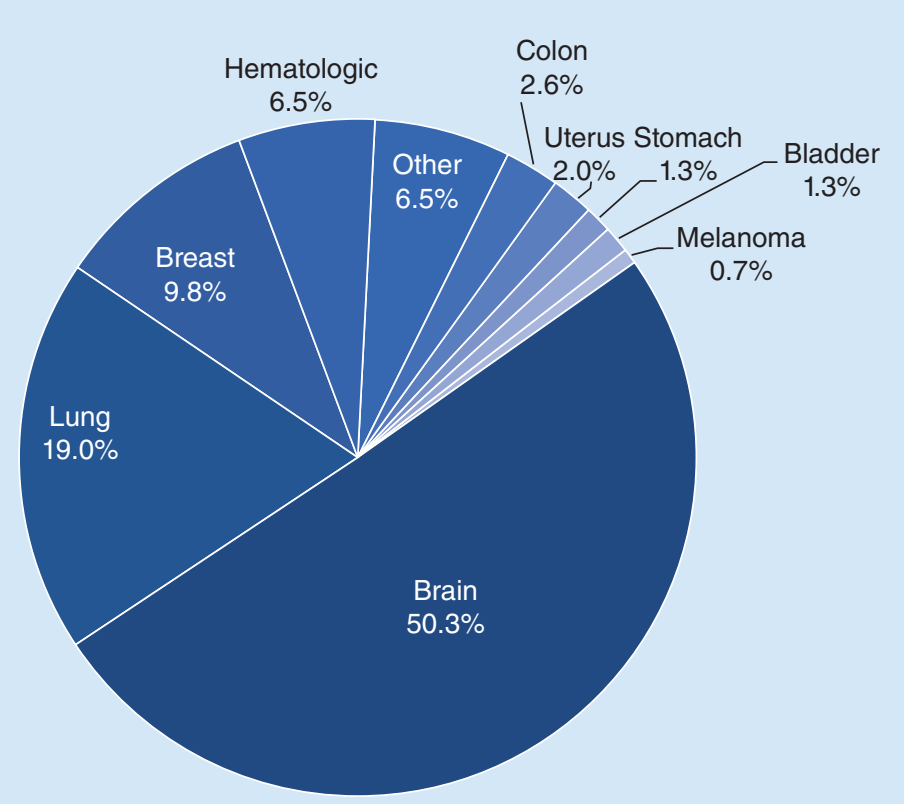

Figure 3. Most common cancer types in our population.

subtypes included colon cancer (2.6\%), uterine cancer (2\%), stomach and bladder cancers (1.3\% each) and melanoma $(0.7 \%)$. Finally, other rare tumors (pancreatic, prostatic, thymic, bone, renal, skin, nasopharyngeal and unknown primary tumors) were also incriminated and constituted together $6.5 \%$.

Tumors most responsible for brain metastases were lung cancer (38.7\%) followed by breast cancer (20\%) and hematological malignancies (13.3\%). Colon cancer accounted for 5.3\% of cases and uterine cancer $4 \%$, followed by stomach and bladder tumors with $2.7 \%$ of cases each, melanoma $1.3 \%$. Other less classical and rare types of brain metastases (cited above) accounted together for $10.7 \%$ of cases. 


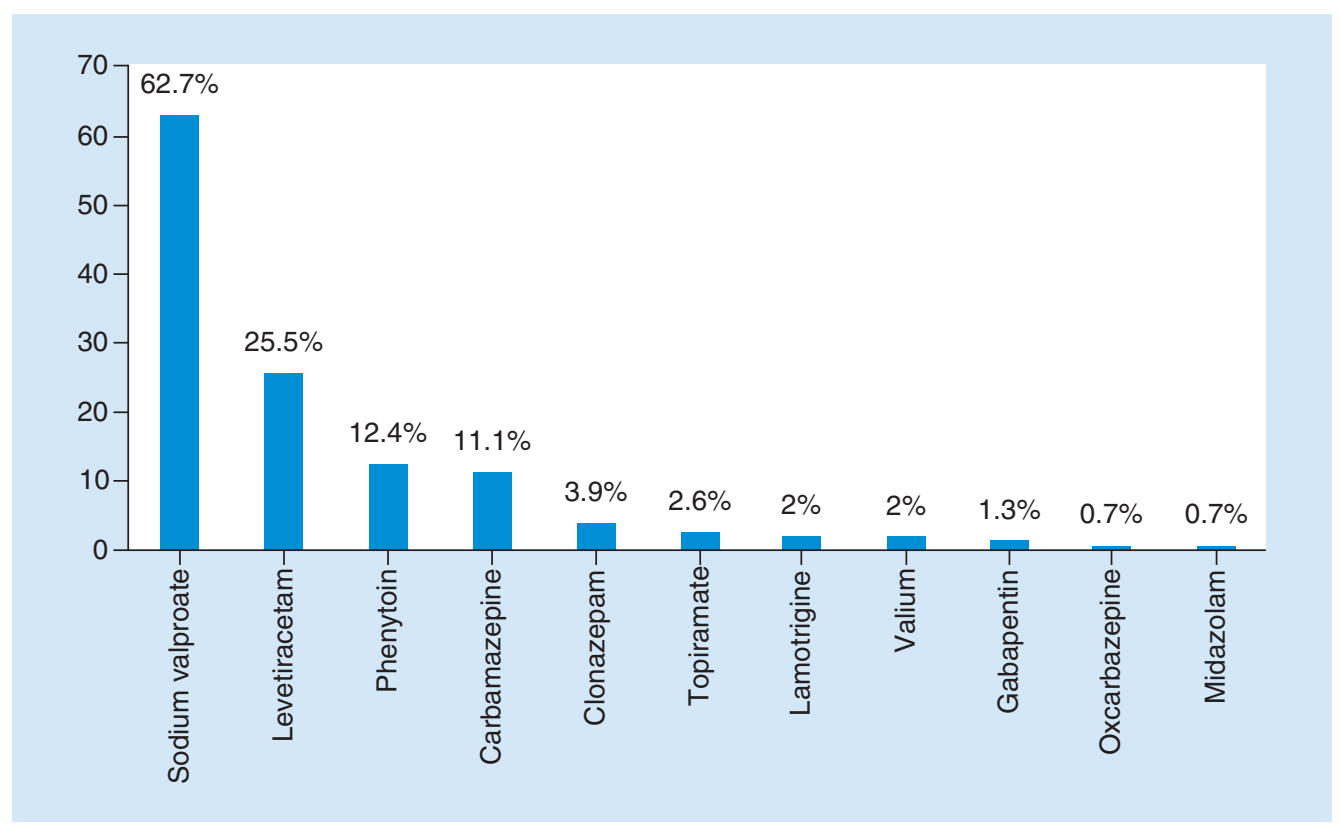

Figure 4. Antiepileptic drugs used in the treatment of seizures among cancer patients.

Among primary brain tumors, malignant (grades III and IV) glial tumors were predominant (96.1\%), followed by medulloblastoma (3.9\%).

Among the AEDs (Figure 4), sodium valproate was the most used (62.7\%), followed by levetiracetam (25.5\%), phenytoin (12.4\%) and carbamazepine (11.1\%). Clonazepam was used in 3.9\% of cases, topiramate in $2.6 \%$, lamotrigine and valium in $2 \%$ of cases each, gabapentin in $1.3 \%$, oxcarbazepine and midazolam in $0.7 \%$ each. Of note, two-thirds of patients were treated by monotherapy, while a third of patients required a shift from one drug to another or a combination of two or more AEDs to control seizures.

\section{Discussion}

In our study, we evaluated the etiologies of seizures in cancer patients. We included metabolic, iatrogenic causes besides the tumor itself. We also evaluated the different types of AEDs used to treat epilepsy in those patients. We found that around $85 \%$ of cases of epilepsy were due to neoplastic involvement of the brain (primary tumors, metastatic tumors or carcinomatous meningitis), which is concordant with previous reports of epilepsy in cancer patients where cerebral lesions were the most common cause of seizures, especially primary tumors rather than metastatic lesions [13].

Among the other etiologies, around 5-6\% of cases were caused by reversible factors such as metabolic disturbances, drugs (e.g., carbapenem) and chemotherapy. Similar reports are seen in the literature where at least $4 \%$ of patients having an extracranial tumor without brain metastases had seizures, commonly due to electrolyte abnormalities or organ dysfunction leading to metabolic encephalopathies, or toxic encephalopathies secondary to chemotherapy or other drugs (antidepressants, neuroleptic agents, antibiotics, commonly used among cancer patients) [14]. This outlines the importance of the monitoring of cancer patients for any metabolic derangement, as well as drug tailoring for every patient in order to prevent and control seizures in this group of patients with reversible factors.

In addition to primary brain tumors, lung and breast cancers were the most common tumors giving rise to brain metastases and were frequently responsible for seizures in 19 and $10 \%$ of patients, respectively. Moreover, melanoma is seldom represented in our cohort due to the small sample size of patients with metastatic melanoma, but it represents the tumor with the highest epileptogenic potential followed by lung then breast cancers, as it appeared in our series, although rates are different from what was previously reported, due to difference in populations and sample sizes [15].

Cancer patients that presented with seizure in our center were usually treated with monotherapy using sodium valproate or levetiracteam in two-thirds of cases. Sodium valproate is suggested because of its experienced efficacy, intravenous availability and both its anticonvulsant and antineoplastic effects [16,17], whereas the use of levetiracetam 
is growing because of its major efficiency with less interactions in cancer patients under chemotherapy. Sodium valproate was the most frequently used anti-epileptic drug in our series, which is in analogy with reported data suggesting a lower rate of recurrent seizures with this molecule (44\%) compared with phenytoin (51\%) and carbamazepine (70\%) [18].

However, in around a third of patients, monotherapy alone was not sufficient to control symptoms despite increasing the dosage; therefore, shifting to another AED or combining different drugs together was needed for seizure control. This combination modality was equally used between primary brain tumors and metastatic lesions, highlighting the pharmacological resistance quality of brain tumors which can be resistant to standard AED treatment, thus impacting the quality of life of individuals and requiring appropriate medications and dosages [17]. This pharmacological resistance has been described in around 20\% of patients with generalized epilepsy and 35\% of those with partial seizures, in which seizures are so frequent or severe that they limit the activities of daily living despite the use of multiple AEDs at the recommended dosage. Multidrug-resistance proteins associated with brain tumors are a major cause of refractoriness; also genetic causes or structural brain lesions are possible explanation for cases of refractory epilepsy [19].

Furthermore, levetiracetam was used in around one quarter of our patients, noting the importance of this molecule that is being frequently used as an add-on therapy when first-line options become insufficient (valproic acid, lamotrigine and topiramate), or when more potency is needed, since some clinical trials demonstrated the superiority of the combination of valproic acid and levetiracetam compared with either drug alone [9].

This study helps better characterize the etiologies of seizures among oncology patients to allow an appropriate management and better prevention of reversible causes.

\section{Future perspective}

With the rising incidence of cancer and the development of new anticancer drugs, patients are living longer and thus, are at risk of developing more brain metastases and epileptic seizures, and since seizures can impair the quality of life, a tight control for epileptic crisis should be implemented. Newer anti-epileptic drugs need to be developed that are more potent on tumor-induced seizures, with less drug-drug interactions and a better safety profile. Multidisciplinary teams are needed in the management of seizures using various drugs, radiation therapy and even surgery, and anti-epileptic prophylactic protocols should be assessed to determine the situations in which they could be used as a primary prophylaxis.

Executive summary

- Cancer patients are at risk of seizures for multiple causes either related or unrelated to the tumor itself.

- Middle-aged men are mostly affected.

- Primary brain tumors and CNS metastases represent major causes of seizures in this population ( $80 \%)$.

- Cerebral metastases originate mostly from the lungs and breasts and are less epileptogenic than primary brain tumors.

- Numerous reversible etiologies are implicated (drugs and metabolic) and can be effectively treated or prevented.

- Two-thirds of the seizures were controlled by monotherapy; whereas in a third of patients, monotherapy was not sufficient, highlighting that tumor-associated seizures are frequently refractory to treatment.

- Among antiepileptic drugs, sodium valproate and levetiracetam were the most used. Less than $1 \%$ of the seizures required sedation by midazolam.

- Seizures impact the quality of life of cancer patients and need an appropriate management but also a prevention for reversible causes.

Financial \& competing interests disclosure

The authors have no relevant affiliations or financial involvement with any organization or entity with a financial interest in or financial conflict with the subject matter or materials discussed in the manuscript. This includes employment, consultancies, honoraria, stock ownership or options, expert testimony, grants or patents received or pending, or royalties.

No writing assistance was utilized in the production of this manuscript.

\section{Open access}

This work is licensed under the Creative Commons Attribution-NonCommercial-NoDerivatives 4.0 Unported License. To view a copy of this license, visit: http://creativecommons.org/licenses/by-nc-nd/4.0/ 


\section{References}

Papers of special note have been highlighted as: $\bullet$ of interest

1. Hildebrand J, Lecaille C, Perennes J, Delattre J-Y. Epileptic seizures during follow-up of patients treated for primary brain tumors. Neurology 65(2), 212-215 (2005).

2. Maschio M. Brain tumor-related epilepsy. Curr. Neuropharmacol. 10(2), 124-133 (2012).

3. Singh G, Rees JH, Sander JW. Seizures and epilepsy in oncological practice: causes, course, mechanisms and treatment. J. Neurol. Neurosurg. Psychiatry 78(4), 342-349 (2007).

4. Shaffrey ME,Mut M, Asher AL et al.,, Brain metastases. Curr. Probl. Surg. 41(8), 665-741 (2004).

5. Platta CS, Khuntia D, Mehta MP, Suh JH. Current treatment strategies for brain metastasis and complications from therapeutic techniques: a review of current literature. Am. J. Clin. Oncol. 33(4), 398-407 (2010).

6. Seute T, Leffers P, ten Velde GPM, Twijnstra A. Neurologic disorders in 432 consecutive patients with small cell lung carcinoma. Cancer 100(4), 801-806 (2004)

- Describes the neurological manifestations associated with the small cell histology in lung cancer.

7. Schouten LJ, Rutten J, Huveneers HAM, Twijnstra A. Incidence of brain metastases in a cohort of patients with carcinoma of the breast, colon, kidney, and lung and melanoma. Cancer 94(10), 2698-2705 (2002).

- Reviews the incidence of brain metastases in various tumor types.

8. Tarhini AA, Agarwala SS. Management of brain metastases in patients with melanoma. Curr. Opin. Oncol. 16(2), 161-166 (2004).

9. van Breemen MSM, Wilms EB, Vecht CJ. Epilepsy in patients with brain tumours: epidemiology, mechanisms, and management. Lancet Neurol. 6(5), 421-430 (2007).

- Reviews the epidemiology and treatment of epilepsy in brain tumors.

10. Bhansali A,Banerjee AK, Chanda A et al.,, Radiation-induced brain disorders in patients with pituitary tumours. Australas. Radiol. 48(3), 339-346 (2004).

11. Perucca E. Clinically relevant drug interactions with antiepileptic drugs. Br. J. Clin. Pharmacol. 61(3), 246-255 (2006).

- Shows the drug interactions that are observed between antiepileptic drugs and cancer drugs.

12. Vecht CJ, Wagner GL, Wilms EB. Interactions between antiepileptic and chemotherapeutic drugs. Lancet Neurol. 2(7), 404-409 (2003).

13. Englot DJ, Chang EF, Vecht CJ. Epilepsy and brain tumors. Handb. Clin. Neurol. 134, 267-285 (2016).

14. Bromfield EB. Epilepsy in patients with brain tumors and other cancers. Rev. Neurol. Dis. 1(Suppl. 1), S27-S33 (2004).

15. Clouston PD, DeAngelis LM, Posner JB. The spectrum of neurological disease in patients with systemic cancer. Ann. Neurol. 31(3), 268-273 (1992).

16. Michaelis M, Doerr HW, Cinatl J. Valproic acid as anti-cancer drug. Curr. Pharm. Des. 13(33), 3378-3393 (2007).

17. Kargiotis O, Markoula S, Kyritsis AP. Epilepsy in the cancer patient. Cancer Chemother. Pharmacol. 67(3), 489-501 (2011).

- Reviews various aspects of epilepsy among cancer patients.

18. Wick W, Menn O, Meisner C et al. Pharmacotherapy of epileptic seizures in glioma patients: who, when, why and how long? Onkologie 28(8-9), 391-396 (2005).

19. Devinsky O. Patients with refractory seizures. N. Engl. J. Med. 340(20), 1565-1570 (1999). 